\title{
ORIENTATION OF UAV IMAGE BLOCKS BY SURFACE MATCHING
}

\author{
J. A. Gonçalves ${ }^{1, *}$, N. Jordão ${ }^{2,1}$, A. Pinhal ${ }^{1}$ \\ ${ }^{1}$ University of Porto, Science Faculty, Rua Campo Alegre, 416-007 Porto, Portugal - (jagoncal, apinhal)@fc.up.pt \\ ${ }_{2}^{2}$ Academia Militar, R. Gomes Freire 203, 1169-203 Lisboa - nuno.jordao@ academiamilitar.pt
}

Commission II, WG II/1

KEY WORDS: UAV, 3D Point Cloud, Aerial triangulation, Exterior orientation, Surface matching

\begin{abstract}
:
Topographic maps are produced in Portugal by the Army Geospatial Data Centre, based on a geographical database collected from aerial photography in resolutions from 0.30 to $0.50 \mathrm{~m}$. Each map sheet is revised with an update interval of 10 years or more. Many changes, such as new roads or power lines, would be possible to update with much higher frequency using UAV imagery. Although the nominal scale of the published paper map sheets is 1:25,000, the actual positional accuracy requirement is of 1 meter, which is compatible with larger scales. Exterior orientation parameters obtained by UAV navigation equipment does not have enough accuracy for that. This paper deals with a method to automatically improve the UAV exterior orientation, intended to be compatible with the positional accuracy standards of the geographic database. The method is based on matching a point cloud derived from UAV imagery, without GCPs, with a reference DSM obtained from conventional aerial photographs, which were oriented by standard aerial triangulation. Results allowed to improve the UAV data to a positional accuracy of 1 meter, making it compatible with the requirements established for the geographic database.
\end{abstract}

\section{INTRODUCTION}

Traditional topographic maps of scale 1:25,000 are produced in Portugal by the Army Geospatial Data Centre (CIGeoE, 2019). The revision process is time consuming and in many cases, especially in less populated areas, it may take well more than 10 years. Even in these areas changes may be significant because of forest fires and reforestation, which originate many new roads and paths. Many users, both in professional and leisure field activities are affected by the outdate of information present in maps.

Many times the more relevant changes, such as the construction of new roads, occur in rather small fractions of a map sheet. New flights of conventional aerial photography are not justifiable for relatively small changes. Imagery data collected by unmanned aerial vehicles (UAV) could fulfil many of the updates of the geographic data base, and be used to originate new map sheet editions.

Normally aerial photographs for the full map production at this scale are collected with a ground sampling distance (GSD) between $30 \mathrm{~cm}$ and $50 \mathrm{~cm}$, flying at heights of a few $\mathrm{km}$ and covering large areas. UAVs fly at much lower altitude, acquiring images with GSD of a few $\mathrm{cm}$ and covering small areas. In most of the countries legislation imposes a flying height limitation of $120 \mathrm{~m}$, but higher flights can be made with authorization from the aviation authorities. As an example, with a DJI Phantom 4, flying at heights of 100 or 300 meters, GSD of $3 \mathrm{~cm}$ and $9 \mathrm{~cm}$, respectively, are obtained. Figure 1 shows examples of image samples in these two situations.

It is obvious that even a GSD of $10 \mathrm{~cm}$ is much more than what is needed for 1:25,000 scale, or even larger scales, such as 1:10,000. Anyway the high resolution of the UAV images allow for the detection of details, such as power lines (figure 2), that many times are not clearly detected in lower resolution aerial images.
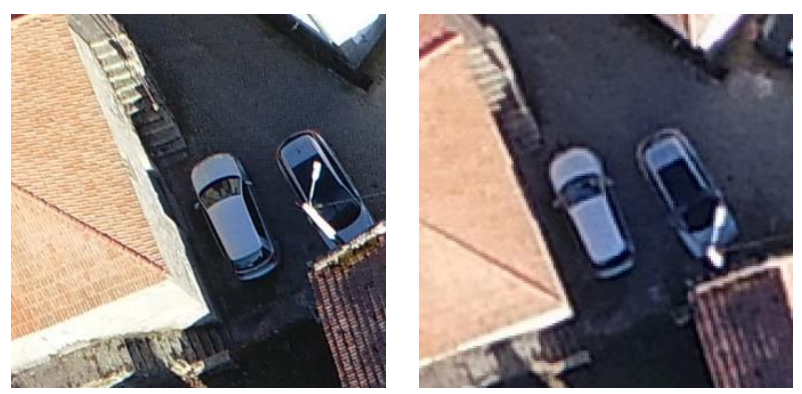

Figure 1. Examples of images acquired from $100 \mathrm{~m}$ height, with GSD $3 \mathrm{~cm}$ (left) and from $300 \mathrm{~m}$, with GSD $9 \mathrm{~cm}$ (right).

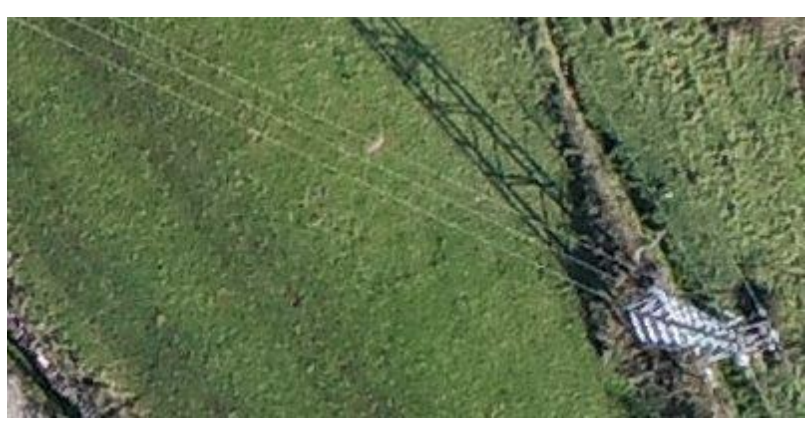

Figure 2. Example of a high voltage pole and lines in the image with GSD of $9 \mathrm{~cm}$.

Due to the very low cost and ease of operation, multi-rotor UAVs, such as the DJI Phantom 4, are being tested for the

* Corresponding author 
purpose of map update and completion. A field user, with a single $\mathrm{UAV}$, and several batteries, can easily acquire more than 5 square $\mathrm{km}$ in 4 hours of operation. Several operators in a few working days can acquire significant amounts of data for the update and completion of the geographic database.

An important problem respects to the need of ground control data for the proper image orientation, at least with the positional accuracy requirements. Frequently, especially in natural environments, artificial signals are used as ground control points (GCPs). This, or in general any extra work of GCP survey, would reduce dramatically the efficiency of a field team. GCP collection should be avoided as much as possible.

UAVs incorporate a pseudo range GNSS receiver for the autonomous navigation. The projection centre coordinates are incorporated in image headers, allowing for an approximate image georeferencing, with a typical uncertainty of a few meters (up to 10 meters), which is not appropriate for the requirements of the geographic database (1 meter).

UAVs equipped with RTK GNSS are now becoming more common (Gerke, and Przybilla), 2016, and would solve the problem. However, their cost is significantly higher and their operation is not so simple, normally requiring the installation of a base station.

The method proposed in this paper relies on using only the standard GNSS navigation positioning of the projection centres. The resulting image exterior orientation parameters need to be improved in order to have an accuracy compatible with the lower resolution images which were the base for the map production. In the conventional aerial triangulation of the aerial images a point cloud is obtained, as well as a digital surface model (DSM), which will act as a reference surface. In the UAV image orientation procedure ("image alignment") a sparse point cloud is obtained, which will have location errors of a few meters, because of the lack of GCPs. This point cloud is adjusted in order to fit the reference DSM.

Figure 3 shows a representation of a portion of the reference DSM and some points of the sparse point cloud. There is a horizontal and a vertical displacement. In fact, a 7 parameter conformal transformation, comprising shift, rotation and scale, could be considered. However, in a small patch, rotation and scale will be negligible because there are no systematic trends in the positions of the projection centres.

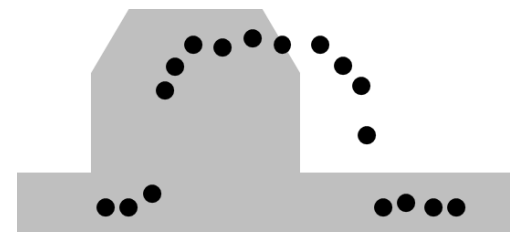

Figure 3. Representation of a DSM (a building), in grey, and a set of points of the same objects, from the sparse cloud.

Surface matching methods are widely used in several photogrammetry operations, such as for example the adjustment of LiDAR strips and other adjustments (Gruen and Acka, 2005, Habib et al., 2008). The method described below was used by Gonçalves (2010) and Gonçalves and Marçal (2007) in the improvement of satellite image orientation. A similar method has also been used by Aguilar et al., 20120, in a process improving the georeferencing of DEM derived from historical aerial photographs.

\section{SURFACE MATCHING METHODOLOGY}

Small patches from the sparse cloud, with variable terrain height, are shifted in order to the fit the reference surface, according to the least squares principle, minimizing the sum of the square of height differences between points and the surface.

For a given DSM grid, heights $\left(Z^{d s m}\right)$ can be estimated for a given location $(X, Y)$, using bilinear interpolation (function DSM, in expression (1)):

$$
Z^{d s m}=\operatorname{DSM}(X, Y)
$$

For a given point of the point cloud, $\left(X_{i}, Y_{i}, Z_{i}\right)$, an horizontal adjustment, $(a, b)$, can be done. A new height is calculated and a residual is obtained as:

$$
\Delta Z_{i}=Z_{i}-\operatorname{DSM}\left(X_{i}+a, Y_{i}+b\right)
$$

For the $n$ points of the point cloud, we can calculate the sum of the squares of the residuals, which is a function of the shift applied.

$$
F(a, b)=\sum_{i=1}^{n} \Delta Z_{i}^{2}
$$

The problem is to determine the shift that minimizes function $F$. This problem can be solved numerically by an iterative process. An interval $\left[\left(a_{\min }, b_{\min }\right),\left(a_{\max }, b_{\max }\right)\right]$ containing the desired shift, is subdivided in a regular grid, function $\mathrm{F}$ is calculated for all grid nodes, and the minimum value is found. The process is repeated with a finer grid around that the first minimum point. The process is repeated until the variation between consecutive iterations is negligible.

Once the minimum is obtained, a vertical shift of the average of the height differences can be applied to the point cloud, in order to make the vertical fit. Figure 4 contains the graphical representation of function $\mathrm{F}$, with contours, with lighter grey for the maximum, value and starting the iterative search for the minimum with intervals $[-10,10]$, both for $\mathrm{X}$ and $\mathrm{Y}$ directions.

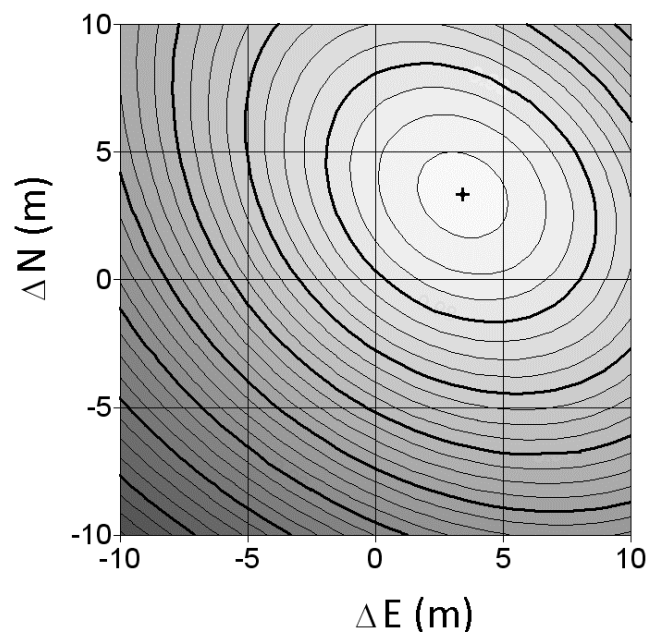

Figure 4. Graphical representation of function F, with contours. The iterative process $\mathrm{w}$. 
This procedure is applied to a small patch of the point cloud. A point of the point cloud patch, corrected by this shift, together with its image coordinates in the images were it appears, can be considered as a simulated GCP. A set of these points, obtained with patches along all the image block can be used to repeat the bundle adjustment and refine the image orientation.

\section{TEST OF THE METHODOLOGY}

The process was tested in a rural area, where the topographic map is out of date for more than 10 years. A highway connecting the cities of Porto and Bragança, crossing the study area, was built several years ago and is not yet represented in the map sheet of the area.

\subsection{Conventional aerial photography}

Aerial photos acquired in 2014 by a digital camera Ultracam XP, with a GSD of $50 \mathrm{~cm}$, were available, together with the result of the aerial triangulation. The horizontal and vertical accuracy on a set of check points were around 1 meter (Root mean square error, RMSE), both for planimetry and altimetry.

A DSM was generated with a pixel spacing of 1 meter, using Agisoft Metashape (Agisoft, 2018). A shaded relief of the DSM is represented in figure 5 , in an extension of $6.0 \mathrm{~km}$ by $2.5 \mathrm{~km}$. The height range in the area was from $610 \mathrm{~m}$ to $800 \mathrm{~m}$.

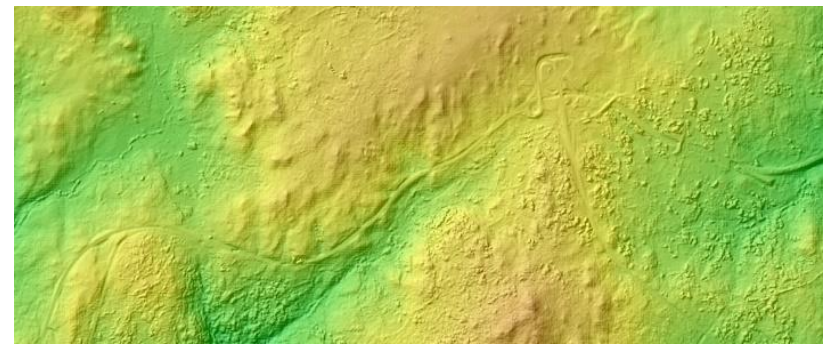

Figure 5. Representation of the DSM with pixel size of 1 meter obtained from aerial photographs.

There is a time difference of 4 years between the aerial images and the UAV images. Changes in vegetation and buildings may originate differences in the DSMs that may pose some difficulties for the surface matching. In general that did not happen in the tests carried out.

\subsection{UAV data acquisition}

A corridor along the new highway, with and extension of $6 \mathrm{~km}$ and width of $700 \mathrm{~m}$ was chosen. A total of more than 2000 images of GSD $4 \mathrm{~cm}$ were acquired with a Phantom 4 Pro, along the study area. Overlaps of $80 \%$ and $70 \%$ were considered for the forward and side overlaps, respectively. This was done in 6 flights of 20 minutes, in a total of approximately 3 hours, changing batteries between flights and moving to new launch points.

Images were oriented in Agisoft Metashape, first without any GCPs, relying only on the projection centres included in the image metadata. A dense point cloud, a DSM (DSM1) and an ortho-mosaic (OM1) were obtained. Then GCPs were introduced in the process, in order to verify the accuracy of the initially obtained datasets. A set of artificial points were placed on the ground before the flight. Together with some painted marks on the roads, a total of 41 points were considered throughout the area. They were surveyed with differential RTK GNSS, with accuracy of 1-2 cm, using a national permanent station network. The bundle adjustment was repeated, yielding residuals on the GCPs with a RMSE of $3.5 \mathrm{~cm}$.

Camera self-calibration was not considered in both cases (without and with GCPs) because a set of interior orientation parameters, obtained in previous calibration works, was available. A new DSM (DSM2) and an ortho-mosaic (OM2) were also extracted.

For the assessment of horizontal and vertical errors a set of 32 points, with a regular distribution along the area were chosen. Horizontal errors were assessed by comparing coordinates of points on ortho-mosaics OM1 and OM2. Errors were relatively small, with the norm around 3 meters and slight variations of direction along the area. Vertical errors were assessed by comparing heights estimated from DSM1 and DSM2 on the 32 check points. They were much larger, with an average of 55 meters. Table 1 contains statistics of the errors in $\mathrm{X}, \mathrm{Y}$, norm $(\mathrm{XY})$ and $\mathrm{Z}$, in meters.

\begin{tabular}{lcccc}
\hline & $e_{X}$ & $e_{Y}$ & $e_{X Y}$ & $e_{Z}$ \\
\hline Minimum & -3.43 & -2.49 & 1.21 & 44.5 \\
Maximum & -1.21 & 2.43 & 3.73 & 67.9 \\
RMSE & 2.18 & 1.91 & 2.90 & 56.1 \\
\hline
\end{tabular}

Table 1. Statistics of horizontal errors (X, Y and norm) of OM1 and vertical errors of DSM1, all in meters .

Graphical representations of the error vectors are shown in figure 6. In the horizontal case (top image), the error vectors are represented with a scale factor 100 . In the vertical case errors are represented in the $\mathrm{Y}$ direction, with a scale factor of 25 . In the case of height, the average ( 55 meters) was removed. The base image in the figures are the orthomosaic obtained with the UAV, with an extension of $5.5 \mathrm{~km}$ in easting.

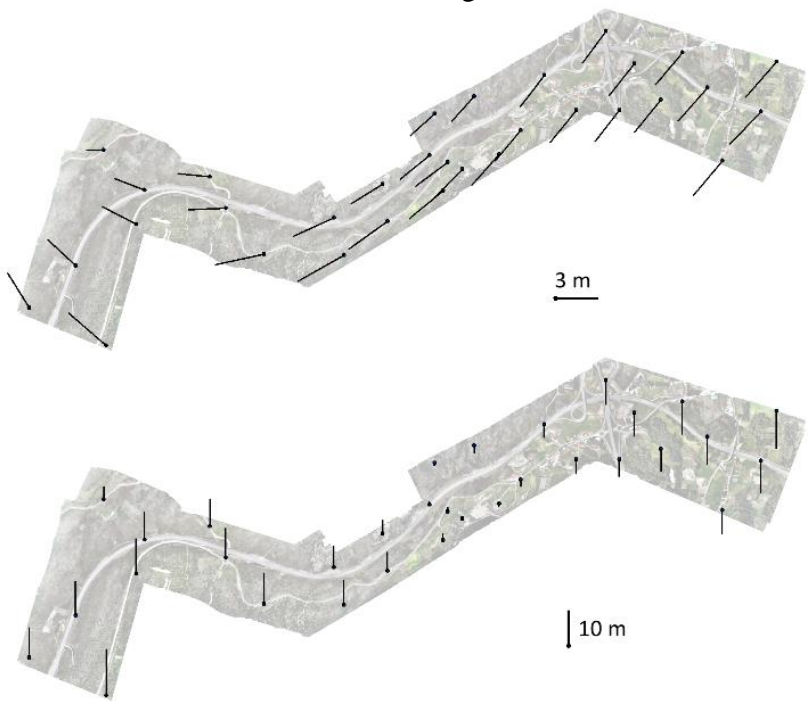

Figure 6. Graphical representation of horizontal (top) and vertical (bottom) errors of datasets OM1 and DSM1. Arrows showing reference error vectors of dimension $3 \mathrm{~m}$ and $10 \mathrm{~m}$, have scale factors of, respectively, 100 and 25 .

\subsection{Surface matching}

The planimetric error is small ( 3 meters) but still larger than the requirement. The proposed method was applied with the 
reference DSM derived from aerial images, which was described before. The point cloud obtained from the UAV, without GCPs, was adjusted to this reference DSM, using the surface matching, in small patches of points in circular areas with a constant radius, containing terrain, vegetation or buildings. These areas must be chosen with some criteria: there has to be height variation (the method simply does not work in flat areas) and slopes must exist in different directions (the method simply does not work if the terrain is a tilted plane).

A total of 8 circular patches were considered, with a radius of 200 meters, which are shown in figure 6 . The choice for $200 \mathrm{~m}$ radius resulted from some empirical testing. Circles with radius $100 \mathrm{~m}$ did not have, in some cases, enough height variation and did not produce so good results.

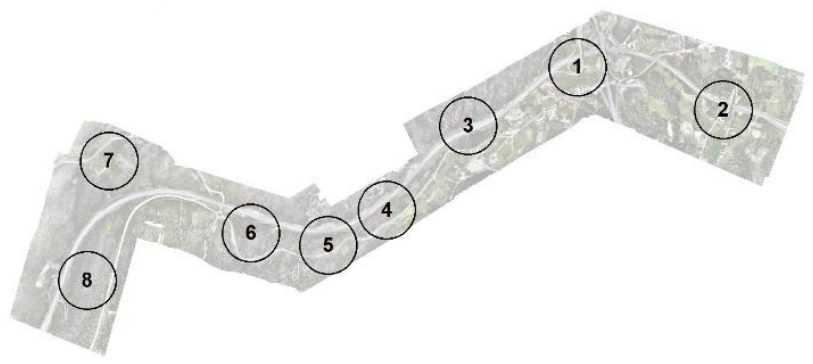

Figure 7. Location of the 8 patches for matching the UAV point cloud with the DSM.

Local horizontal and vertical shifts were obtained, which are listed in table 2. Using the error vectors shown in Figure 5, the expected shifts were determined and compared with the ones obtained by surface matching. Differences are listed in table 2 .

The RMSE of the shifts found and the of the differences are also listed in table 2.

\begin{tabular}{|c|rrr|rrr|}
\hline \multirow{3}{*}{ Patch } & \multicolumn{3}{|c|}{$\begin{array}{c}\text { Shifts found by } \\
\text { surface matching }\end{array}$} & \multicolumn{3}{c|}{$\begin{array}{c}\text { Difference to } \\
\text { expected shifts }\end{array}$} \\
\cline { 2 - 7 } & $\mathrm{dX}$ & $\mathrm{dY}$ & $\mathrm{dZ}$ & $\mathrm{e}$ & $\mathrm{e}$ & $\mathrm{e}_{\mathrm{Z}}$ \\
\hline 1 & -1.9 & -3.7 & 49.1 & -0.2 & -1.4 & 0.7 \\
2 & -3.1 & -1.0 & 47.1 & -1.1 & 1.4 & -0.3 \\
3 & -0.3 & -2.1 & 52.9 & 1.3 & -0.3 & 0.0 \\
4 & -3.9 & -3.0 & 58.4 & -1.1 & -1.0 & -1.9 \\
5 & -1.6 & -1.6 & 61.0 & 1.7 & 0.2 & -0.7 \\
6 & -4.1 & -2.0 & 60.8 & -0.7 & -1.3 & -2.8 \\
7 & -1.4 & 0.5 & 62.9 & -0.2 & 0.5 & 1.8 \\
8 & -2.6 & 0.7 & 62.6 & -0.6 & -1.1 & -1.9 \\
\hline RMSE & 2.65 & 2.11 & 57.16 & 0.98 & 1.02 & 1.56 \\
\hline
\end{tabular}

Table 2. Shifts found by matching, differences to the shifts measured manually,

The horizontal location error of the UAV point cloud, of around 3 meters, was corrected with an accuracy of 1 meter. In fact, even if the initial mis-location were larger (e.g. 10 meters) the same result would be obtained.

The vertical location error of the UAV point cloud, much larger, and with systematic trends along the area, was also corrected, with a slightly lower accuracy, of 1.5 meters.

Obviously, the proposed method is not able to achieve the same positional accuracy in the orthomosaic and in the DSM as the ones obtained with GCPs. Anyway, the reference surface allowed for an UAV image orientation improvement, leading to positional errors of the order of the reference surface accuracy. That is enough to derive data compatible with the medium to large scales involved in the generation of the geographic database for topographic map update.

\section{DISCUSSION AND CONCLUSIONS}

Local updates of topographic maps of scale 1:25,000 are being done, using UAV images, not with the intention of revising full map sheets, but essentially areas, mainly along corridors, with more significant changes.

Although there is a significant difference between UAV image resolution and the aerial photography normally used for this scale, some efficiency in UAV image acquisition can be obtained, especially if field teams do not need to collect GCPs for image orientation.

UAV image orientation is possible with the method described, based on the matching of an UAV point cloud and a reference DSM surface derived from the initial aerial photos. It was possible to achieve a positional accuracy, slightly better in planimetry than in altimetry, that is compatible with the established requirements for the geographic database that is used for the map update.

Future improvements will consider the automation of the process, with automatic choice of the parts of the point cloud more appropriate to make the adjustment to the reference surface. In order to have an accurate planimetric surface matching, slopes must exist in different directions. That can be evaluated from a slope and aspect rasters derived from a digital elevation model of the area, for example the one that resulted from the aerial photographs.

\section{ACKNOWLEDGEMENTS}

To DGT (Direcção Geral do Território) for the use of the GNSS permanent stations. To the $\mathrm{CIGeoE}$ (Centro de Informação Geoespacial do Exército) for the provision of aerial photographs and associated data.

\section{REFERENCES}

Agisoft, 2018. Agisoft Metashape User Manual - Professional Edition, Version 1.5. Available in:

https://www.agisoft.com/pdf/metashape-pro_1_5_en.pdf

Aguilar, F.J., Aguilar, M. A., Fernández, I., Negreiros, J. G., Delgado, J., and Pérez, J. L., 2012. A New Two-Step Robust Surface Matching Approach for Three-Dimensional Georeferencing of Historical Digital Elevation Models. IEEE Geoscience and remote Sensing Letters, Vol. 9, no. 4, July 2012.

CIGeoE, 2013. Web pages of the Portuguese Centre for Geospatial Information of the Army. http://www.igeoe.pt. Accessed in March 2019.

Gerke, M. and Przybilla, H.-J., 2016. Accuracy Analysis of Photogrammetric UAV Image Blocks: Influence of Onboard RTKGNSS and Cross Flight Patterns, Photogrammetrie Fernerkundung - Geoinformation (PFG), 2016 (1), 17-30. DOI: $10.1127 / \mathrm{pfg} / 2016 / 0284$ 
Gonçalves, J.A., 2010. Automatic image orientation and DSM extraction from ALOS-PRISM triplet images. IEEE IGARSS 2010 Proceedings, Honolulu, USA, 2295-2298 (doi: 10.1109/IGARSS.2010.5652476).

Gonçalves, J.A., Marçal, A.R.S., 2007. Automatic Orthorectification of ASTER Images by Matching Digital Elevation Models, Springer Lecture Notes in Computer Science, Vol. 4633 (ICIAR 2007), 1265-1275.

Gruen, A., Akca, D., 2005. Least squares 3D surface and curve matching. ISPRS Journal of Photogrammetry and Remote Sensing 59 (3), 2005: 151-174.

Habib, A. F., Kersting, A. P., Ruifanga, Z., Al-Durgham, M., Kim, C., Lee, D. C., 2008. Lidar strip adjustment using conjugate linear features in overlapping strips. The International Archives of the Photogrammetry, Remote Sensing and Spatial Information Sciences. Vol. XXXVII. Part B1. Beijing 2008. P. 385-390. 